\title{
Implementing Closed-Loop Control in an Engineering Design Process in the Presence of Uncertainties
}

\author{
George Platanitis and Remon Pop-Iliev \\ University of Ontario Institute of Technology \\ george.platanitis@uoit.ca \\ remon.pop-iliev@uoit.ca
}

\begin{abstract}
Much research has been accomplished in the last several years in trying to describe the dynamics of a complex design process in a quantitative, meaningful manner. Such methodologies have recently been implemented in an academic setting, where there is a need for instructors developing their own design projects to have a meaningful method of quantifying the relative difficulty and feasibility of completion of such project in a limited time academic term. Upper year projects $\left(2^{\text {nd }}, 3^{\text {rd }}\right.$, and $4^{\text {th }}$ year) are often quite complex, multidisciplinary, and consist of many tasks with complex relationships which must be performed concurrently. One useful method that describes task relationships is the Design Structure Matrix (DSM), which along with its extension, the Work Transformation Matrix (WTM), have proven useful in predicting convergence of design iteration, as well as identifying particularly slow tasks. But design environments are uncertain and can undermine the stability of a design process. Also, even though familiarity with a design project allows the determination of a better dynamic model for the process than for a new project, even this model is not absolute and will contain uncertainties. Therefore, methodologies are needed to determine how resources can be reallocated to expedite slowly converging tasks with uncertainties present. In this paper, closed-loop control will be implemented to quantify and guide the reallocation of resources of a design process containing many coupled, concurrent design tasks to expedite particularly slow ones, as well as a methodology to overcome the uncertain nature of the design process model.
\end{abstract}

\section{Introduction}

Predicting the nature of the dynamics of a design process is often a difficult one, especially where many tasks are involved that are often coupled. While research in recent years has attempted to provide modelling methodologies for design processes, no model can be absolute in predicting the process' behaviour. Many factors can determine the nature of the convergence of the design process, from familiarity and experience with the problem involved to availability of tools and resources.

With complex design projects involving several iterations to achieve a feasible solution, there will often be tasks that are particularly slow, that will hold the design process back as a whole before a solution is finally reached. In such situations, it becomes necessary to redistribute resources among the tasks in such a way as to expedite the slower tasks, and in turn expedite the design process as a whole. How can this redistribution be quantified? And will this redistribution create a more reliable process when there are uncertainties? Using a third-year design project as a case study, a model is developed for the design process and a closed-loop control law is developed to quantify the resource redistribution to expedite tasks when uncertainties in the design process model exist. This information will allow instructors to guide students in systematically allotting resources (time, tools, etc.) to increase the speed of task iteration, as well as decrease the number of iterations required to complete a particular task.

\section{Background}

Methodologies such as Design Structure Matrix/Work Transformation Matrix (DSM/WTM) have been investigated in the last several years to identify task relationships and their strengths; to use as a basis in developing algorithms to reduce task coupling; to predict design convergence; and to determine stability characteristics, identify design modes, and identify particularly slow tasks [1-5]. However, closing the loop in the design process to expedite tasks is an area of recent research. In one example [6], a closed-loop design change control workflow was developed to apply product data management to the conceptual design phase, with the methodology evaluated for a steel foundry. The closed-loop strategy proposed allowed the product planning activities to be involved early in the design process, specifically at the 
conceptual design phase. Using this process, the closed-loop design change control workflow ensures conceptual schemes are continually improved, stock on hand is considered, and manufacturability of the design during the conceptual phase is considered.

In another example [7], closed-loop engineering design was implemented in product lifecycle management (PLM) to provide more flexibility and adaptability with a higher quality level. PLM was studied along the dimensions of time and rigidity, with rationale based on establishing flexible borders for engineering activities and providing online error detection and online compensation reactions. As such, these activities are modified dynamically and adapt themselves in real-time to market requests and technological, as well as resource limitations.

However, design and development processes will have some inherent level of uncertainty, requiring any closed-loop control on the process to be robust, allowing the process to converge. In one example, a multi-objective possibilistic optimization model was developed for a closed-loop supply chain network design for both forward and reverse supply chain networks in the presence of uncertain demands, returns, delivery times, costs, and capacities [8]. The goal of the methodology was to minimize costs, as well as lateness of product delivery. Tactical material flow decisions were also included to avoid suboptimalities resulting from separated design.

One of the few results available in which closed loop control is quantified and applied to a design process is in Lee et al [9]. The authors used DSM methods to model the design process and determined the tasks that consume large, disproportionate amounts of resources and time to complete. They then determined a closed-loop gain matrix based on eigenvalue analysis, defined to improve the stability

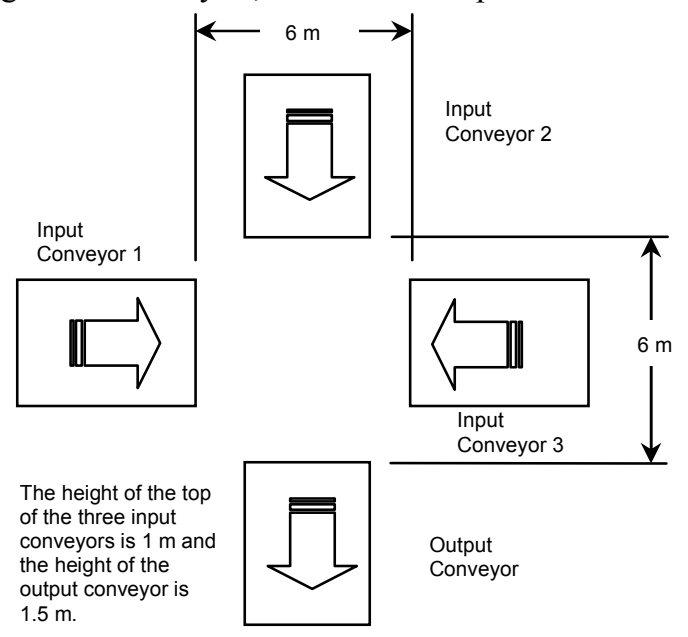

Figure 1. Conveyor belt layout and scaling pertaining to tire manipulator design project. and convergence rate of design tasks, as well as to guide resource distribution to expedite particularly slow tasks. However, the question remains as to how well the closed-loop process will converge when the design process model contains uncertainties.

This paper examines a case study of a third-year engineering design project at UOIT, for which closedloop control will be applied to provide guidance to students in distributing resources to complete various tasks faster and more efficiently. First, a DSM-WTM model for the design process will be presented and uncertainties will be introduced. Then, a control law will be developed and applied to the design process to help quantify how resources can be used to expedite convergence of various tasks, particularly those that are slow to converge. The reliability of the closedloop process will be evaluated with uncertainties present.

\section{Third-Year Design Project}

Engineering students enrolled in mechanical-based programs at UOIT in the $3^{\text {rd }}$ year undertake an integrated design project between two courses which combine concepts of $\mathrm{CAD} / \mathrm{CAM} / \mathrm{CAE}$ design principles (3D Scanning, Solid Modelling, Rapid Prototyping, Finite Element Methods, etc.) with kinematic and dynamic analysis of machines.

Working in groups of about 4-5, students were charged with the task of designing a mechanism for handling and manipulating three different tire sizes, to be picked up from three input conveyor belts oriented at 90 degrees to each other (Fall 2008 edition), and placed on an output conveyor (see Fig. 1). The following requirements were to be adhered to:

- Factor of safety between 2.5 and 5 (anything greater than 5 is considered over-engineered).

- Able to handle tires of maximum weight of $50 \mathrm{~kg}$ each.

- In addition to the CAD design, students must build and demonstrate a prototype of their design using Lego Mindstorms ${ }^{\circledR}$. Students were required to design the actual manipulator in CAD using real parts and to proper size (not to simply enlarge the Lego pieces).

A suggested task list and order thereof is given below, but it should be noted that tasks C-L will be coupled to some degree:
A. Determine specifications
B. Design concept
C. Design arm
D. Design arm joints
E. Design grip mechanism
F. Design rotating base
G. Finite Element Analysis
H. Dynamic simulation 
I. Factor of safety

J. Failure mode analysis

K. Build prototype

L. Evaluate prototype

Regardless of the complexity of a design project, students often carry out the design effort in a somewhat disjointed order, focusing their efforts and resources on the wrong tasks at the wrong time. Using DSM methodologies, and the closed-loop design process as presented in this paper, students can see more clearly what tasks they should dedicate their efforts and resources to in order to be able to expedite those tasks, reducing the total number of iterations required to complete the design project. This quantified measure of resource usage will also be useful in reducing the number of iterations required to complete a project when there are unexpected events.

\section{DSM-WTM Representation}

Figure 2 shows a WTM representation of the tire and wheel manipulator. This representation not only shows the relationships between tasks, but also the strength of the task dependencies [1-5]. To use this model the following assumptions are necessary [2]:

- All tasks are completed at every stage (fully parallel iteration).

- Rework is a function of work done in the previous iteration stage.

- Work transformation parameters are time invariant.

Simple strength dependency values are assigned to each of the task relationships as follows [2]: 0.5, 0.25, and 0.05 for strong (S), medium (M), and weak (W) dependencies respectively, with the following interpretation [1]:

- Strong - information required from previous task to begin task.

- Medium - information required to end task.

- Weak - information required to check result compatibility.

- Zero - no information required

This dependency can be thought of as the amount of work (in terms of a percentage of time to determine a parameter in the original iteration) that the upstream task creates for a downstream task. For example, if we look at the task dependency defined in box I-F (row I, column F), in Fig. 2, a weak dependency (0.05) is shown. Quantitatively, this means that Task I not only depends on information from Task F, but Task F

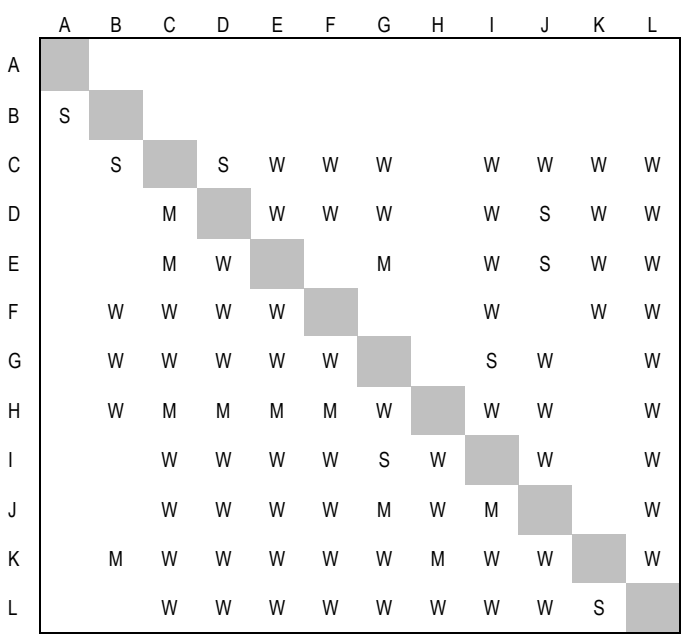

Figure 2. WTM matrix representation for tasks to be performed in tire manipulator design.

will create $5 \%$ rework on Task I at the next iteration stage.

\subsection{Mathematical Formulation}

At each iteration stage, an amount of work will have been completed for each task. The idea of the work vector is introduced here, and the elements of the work vector indicate how much work is left for each task to complete. As the design process progresses from iteration stage $t$, the work remaining at the next stage is given by the discrete state-space equation $[2,5]$ :

$$
\mathbf{u}_{t+1}=\mathbf{A} \mathbf{u}_{t}, \quad t=0,1,2, \ldots, \infty
$$

where $\mathbf{u}_{t}$ is the work vector at stage $t$ and $\mathbf{A}$ contains the strength dependencies of the WTM, where each $a_{i j}$ element $(i \neq j)$ implies that doing a unit of work on task $j$ creates $a_{i j}$ units of work on task $i$. Each element in the work vector represents the work remaining for the task it is associated with at iteration stage $t$. Treating the dynamics of the design process as a discrete-time system is logical [5]. Just as the state variables of a discrete-time system are computed at discrete intervals, the work vector is computed at discrete intervals (the iterations themselves) for the design process model. Note that Eq. 1 is the homogeneous state-space system (that is, the actual response depends on the initial conditions only). In order to include external inputs, Eq. 1 is modified as: 


$$
\mathbf{u}_{t+1}=\mathbf{A} \mathbf{u}_{t}+\mathbf{B}_{1} \boldsymbol{\delta}_{t}+\mathbf{B}_{2} \boldsymbol{\varepsilon}_{t}
$$

where $\mathbf{B}_{1}$ is the Disturbance Transformation Matrix [10], $\boldsymbol{\delta}_{t}$ is the disturbance input (to be used later to represent work added as a result of unexpected events into the design process), $\mathbf{B}_{2}$ is the input matrix (representing the proportion of common resources shared between tasks), and $\boldsymbol{\varepsilon}_{t}$ represents the additional resources required for the design process to converge to a desired state [9]. For simplicity, $\mathbf{B}_{1}=\mathbf{B}_{2}=\mathbf{I}$. In industry applications, additional resources may be acquired through overtime work, adding new staff, recruiting temporary help, outsourcing, etc.

\subsection{Uncertainties in Design Process Model}

With any design process, uncertainties exist, which can change the amount of work remaining at each iteration stage from what the WTM model can predict. Factors which may add uncertainty include competition, technological improvements, and changes to customer requirements. Also, different design groups (as was found with this case study) may determine different models for the design process, resulting in variations in the work remaining in each task at each iteration stage. Figure 3 shows the

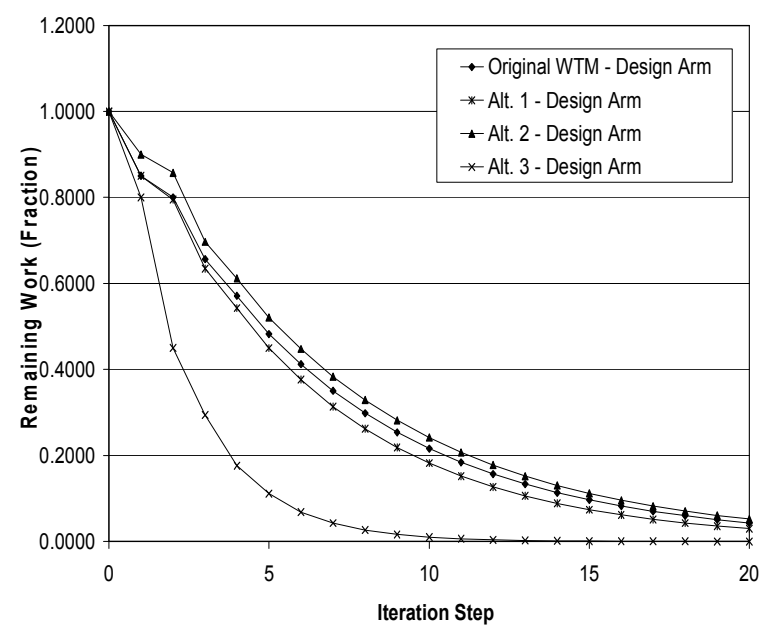

Figure 3. Comparison of task iterations for original and alternative WTM matrices for Task C - Design Arm.

variations in the dynamic responses for different models of the same design process, using the Design Arm task as an example.

To include uncertainties in the design process, a random variation within $\pm 10 \%$ work remaining at each stage is introduced, representing a somewhat more realistic response of the design process at each iteration stage (that may be the result of unknown factors). In the next section, a method for estimating these states will be introduced to be used in the closedloop control of the design process.

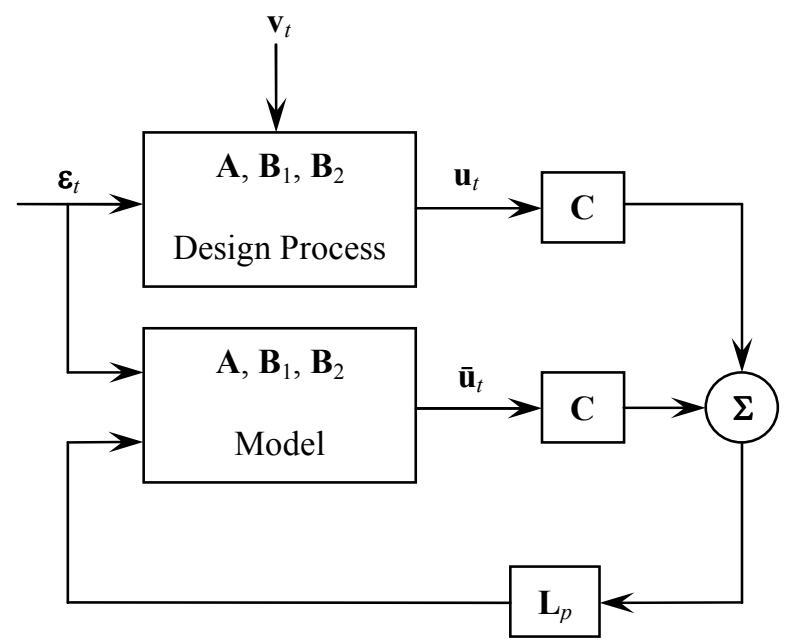

Figure 4. Estimator for tire and wheel manipulator design process.

\subsection{Estimation and Control}

To handle the uncertainty in $\mathbf{u}_{t}$, an estimator $[11,12]$ is introduced to provide estimated values, $\overline{\mathbf{u}}_{t}$, for the remaining work at each stage. The equation for the estimator is:

$$
\overline{\mathbf{u}}_{t+1}=\mathbf{A} \overline{\mathbf{u}}_{t}+\mathbf{B}_{1} \boldsymbol{\delta}_{t}+\mathbf{B}_{2} \boldsymbol{\varepsilon}_{t}+\mathbf{L}_{p}\left[\mathbf{y}_{t}-\mathbf{C} \overline{\mathbf{u}}_{t}\right]
$$

where $\mathbf{L}_{p}$ is the estimator gain matrix, $\mathbf{y}_{t}$ is an output vector $\left(\mathbf{y}_{t}=\mathbf{C} \mathbf{u}_{t}\right.$ ), and $\mathbf{C}$ is an output matrix (and will equal I so that simply $\mathbf{y}_{t}=\mathbf{u}_{t}$ ). Figure 4 shows the estimator scheme. Since this estimator predicts the remaining work vector at the next iteration step, it is called a prediction estimator $[11,12]$. Note that $\mathbf{v}_{t}$ is the random variation in the remaining work for the actual design process.

To utilize closed-loop control in the design process, the following control law is defined:

$$
\boldsymbol{\varepsilon}_{t}=-\mathbf{K} \overline{\mathbf{u}}_{t}
$$

where $\mathbf{K}$ is a feedback gain matrix, whose define the degree of influence that all other tasks have on the resource use of a task [9]. Note from Eq. 4 that the estimated work remaining is used in the feedback loop of the design process (with $\mathbf{u}_{t}$ being uncertain, one should use the best estimate of the work vector at every iteration stage). The matrix $\mathbf{K}$ may be determined by any standard means, but for this purpose, it will be optimally determined using a discrete linear quadratic regulator, where $\mathbf{K}$ is determined such that the following performance index is minimized [12]: 


$$
J=\frac{1}{2} \sum_{t=0}^{\infty}\left[\mathbf{u}_{t}^{T} \mathbf{Q} \mathbf{u}_{t}+\boldsymbol{\varepsilon}_{t}^{T} \mathbf{R} \boldsymbol{\varepsilon}_{t}\right]
$$

where $\mathbf{Q}$ and $\mathbf{R}$ are chosen to be positive definite matrices, with values chosen to balance state errors $\mathbf{u}_{t}^{T} \mathbf{u}_{t}$ and control usage $\boldsymbol{\varepsilon}_{t}^{T} \boldsymbol{\varepsilon}_{t}$. Typically, $\mathbf{Q}$ is a diagonal matrix with one or more weighting factors on the state vector elements, and $\mathbf{R}=\mathbf{I}$, where $\mathbf{I}$ is the identity matrix. In this investigation, Tasks C-F will be weighted (that is, weight values will be introduced to the elements $q_{11}, q_{22}, q_{33}$, and $q_{44}$ in $\mathbf{Q}$ ) in deriving the closed-loop gain matrix since they will have disturbances introduced to them, and therefore, these tasks will need to be expedited so that the design process will converge quickly.

Table 1. Iterations to Convergence with $\pm 10 \%$ Random Variation in Remaining Work.

\begin{tabular}{|l|c|c|c|}
\hline & $\begin{array}{l}\text { No } \\
\text { Dist. }\end{array}$ & $\begin{array}{l}\text { Dist. At 5 } \\
\text { It. (100\% } \\
\text { More Work) }\end{array}$ & $\begin{array}{l}\text { Dist. At 10 } \\
\text { It. (100\% } \\
\text { More Work) }\end{array}$ \\
\hline $\begin{array}{l}\text { Avg. } \\
\text { \#It. }\end{array}$ & 19 & 21 & 24 \\
\hline $\begin{array}{l}\text { Range } \\
\text { of It. }\end{array}$ & $17-25$ & $19-25$ & $21-26$ \\
\hline
\end{tabular}

\subsection{Results}

Before implementing the closed-loop control, some results are presented in Table 1 to illustrate the variation in convergence of the design process when the model is uncertain and when no control is present. The average number of iterations required for convergence is shown, as well as the range between highest and lowest number of iterations required for several simulation runs. Variations of $\pm 10 \%$ work remaining were introduced at each iteration stage to provide some uncertainty in the predictions of the model. Unexpected events (disturbances) were introduced at the $5^{\text {th }}$ and $10^{\text {th }}$ iteration by imposing 100 percent more work for Tasks $\mathrm{C}-\mathrm{F}$ at these iteration stages.

To determine the closed-loop gain matrix, weight values of $q_{i i}=0.1$ (where $i=1, \ldots, 4$ ) were used, yielding the following optimal $\mathbf{K}$ :

$\mathbf{K}=$

$\left[\begin{array}{llllllllll}0.0051 & 0.0553 & 0.0087 & 0.0085 & 0.0174 & 0.0030 & 0.0188 & 0.0105 & 0.0078 & 0.0083 \\ 0.0328 & 0.0052 & 0.0088 & 0.0086 & 0.0158 & 0.0024 & 0.0150 & 0.0620 & 0.0089 & 0.0084 \\ 0.0257 & 0.0075 & 0.0017 & 0.0016 & 0.0278 & 0.0011 & 0.0091 & 0.0497 & 0.0061 & 0.0060 \\ 0.0073 & 0.0073 & 0.0061 & 0.0015 & 0.0048 & 0.0010 & 0.0089 & 0.0039 & 0.0059 & 0.0059 \\ 0.0089 & 0.0111 & 0.0059 & 0.0057 & 0.0173 & 0.0037 & 0.0186 & 0.0117 & 0.0047 & 0.0052 \\ 0.0016 & 0.0016 & 0.0010 & 0.009 & 0.0028 & 0.0006 & 0.0026 & 0.0021 & 0.0008 & 0.0008 \\ 0.0085 & 0.0087 & 0.0051 & 0.0049 & 0.0155 & 0.0031 & 0.0141 & 0.0116 & 0.0043 & 0.0044 \\ 0.0093 & 0.0190 & 0.0076 & 0.0074 & 0.0238 & 0.0056 & 0.0275 & 0.0106 & 0.0059 & 0.0071 \\ 0.0031 & 0.0036 & 0.0019 & 0.0018 & 0.0056 & 0.0013 & 0.0056 & 0.0043 & 0.0017 & 0.0017 \\ 0.0032 & 0.0037 & 0.0019 & 0.0019 & 0.0058 & 0.0013 & 0.0057 & 0.0045 & 0.0017 & 0.0017\end{array}\right]$
As an example, in the second row of the first column (Task row D, Task column C), the value 0.0328 indicates that when Task $\mathrm{C}$ has 100 percent work remaining at some stage $t$, Task $\mathrm{D}$ will require $3.28 \%$ more of its own resources to do the additional work to help expedite Task C.

With feedback control, the number of iterations required for the design process to converge is reduced. Table 2 shows the number of iterations to convergence under the same circumstances. It should be noted that the use of closed-loop control occurs at the onset of the design process when no disturbances were present (thus reducing the number of iterations required for the design process to converge), as well as at the onset of disturbances (such that tasks are expedited to mitigate the effect of disturbances and prevent unnecessary iterations).

Table 2. Iterations to Convergence with Closed-Loop Control Imposed.

\begin{tabular}{|l|l|l|c|}
\hline & $\begin{array}{l}\text { No } \\
\text { Dist. }\end{array}$ & $\begin{array}{l}\text { Dist. At 5 } \\
\text { It. (100\% } \\
\text { More Work) }\end{array}$ & $\begin{array}{l}\text { Dist. At 10 } \\
\text { It. (100\% } \\
\text { More Work) }\end{array}$ \\
\hline $\begin{array}{l}\text { Avg. } \\
\text { \#It. }\end{array}$ & 11 & 16 & 20 \\
\hline $\begin{array}{l}\text { Range } \\
\text { of It. }\end{array}$ & $10-14$ & $13-18$ & $18-21$ \\
\hline
\end{tabular}

To interpret the results of the control input, the case where a disturbance occurred at the $5^{\text {th }}$ iteration will be examined as an example. At the point when the disturbance occurred, the control input is:

$$
\begin{aligned}
& \boldsymbol{\varepsilon}_{5}=-\mathbf{K} \overline{\mathbf{u}}_{5} \\
& =-\left[\begin{array}{llllllllll}
0.1458 & 0.1238 & 0.0915 & 0.0467 & 0.0720 & 0.0113 & 0.0618 & 0.0982 & 0.0237 & 0.0244
\end{array}\right]^{T}
\end{aligned}
$$

The elements of $\boldsymbol{\varepsilon}_{5}$ denote how many more resources or extra work is required for each task at the $5^{\text {th }}$ iteration stage to mitigate the effect of the disturbance. For Tasks C-F, at the onset of the disturbance, the amount of extra resources that must be allocated to these tasks so that the design process converges within $\sim 16$ iterations is $14.58 \%, 12.38 \%, 4.67 \%$, and $7.20 \%$ respectively. The negative sign indicates that extra work or resources are needed. At every iteration stage from this point onward, the $\boldsymbol{\varepsilon}_{t}$ vector indicates how resources should be allotted to expedite the design process to mitigate disturbance effects. For example, the resource allocation at the $10^{\text {th }}$ stage of iteration will be given by: 
$\boldsymbol{\varepsilon}_{10}=-\mathbf{K} \overline{\mathbf{u}}_{10}$

$=-\left[\begin{array}{llllllllll}0.0284 & 0.0329 & 0.0267 & 0.0106 & 0.0178 & 0.0028 & 0.0154 & 0.0237 & 0.0059 & 0.0061\end{array}\right]^{T}$

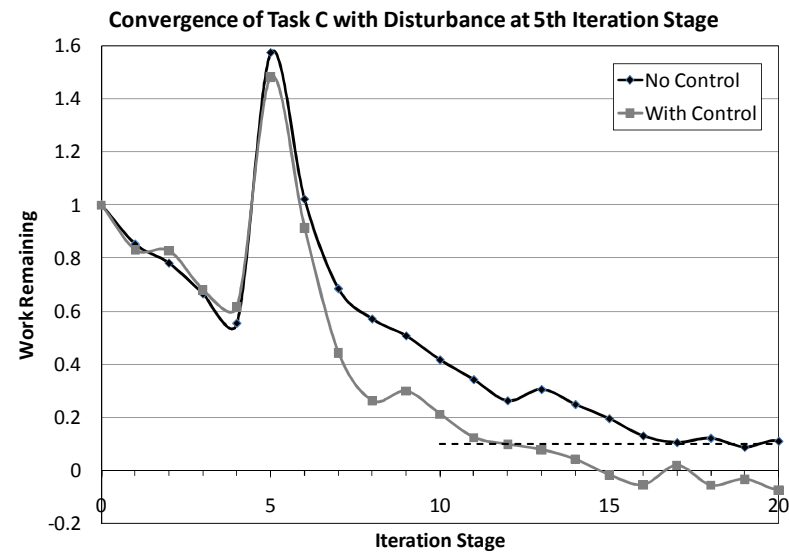

Figure 5. Convergence of Task $\mathrm{C}$ with and without closed-loop control.

By this stage, Tasks C-F will only need $2.84 \%, 3.29 \%$, $2.67 \%$, and $1.06 \%$ extra resources to expedite these tasks (and consequently, the rest of the design process). Figure 5 illustrates the convergence of one of the tasks (Task C) with and without closed-loop control in the presence of random variations in work remaining at each iteration stage in mitigating a disturbance/unexpected event. The dashed line indicates where 10 percent work remaining is. It can be seen that without control, Task $\mathrm{C}$ converges after 19 iterations, while convergence occurs after 13 iterations with control (after this point, work remaining can be ignored, since the task has essentially converged).

\section{Conclusions}

A mathematical model for the design process of a third-year engineering design project at UOIT was developed to handle uncertainties. This was accomplished by using an estimator to provide a best guess of the work remaining at each iteration stage. Using this model, a closed-loop control was developed to quantify resource allocation to expedite tasks when work remaining at each stage is uncertain, as well as to mitigate the effects of unexpected events or disturbances in the process. Closed-loop control in the design process assisted in quantifying resource use at each iteration stage to speed up convergence of the design process and may serve as a useful guide to students and instructors in assisting them with design task planning and providing a priori knowledge of how to efficiently use resources.

\section{Acknowledgements}

The authors would like to thank the Natural Sciences and Engineering Research Council (NSERC) of Canada, General Motors Canada Limited (GMCL), and UOIT for financially supporting UOIT's Design Chair budget, part of which supported this research work. The authors are also grateful for the hardware and software provided by PACE (Partners for the Advancement of Collaborative Engineering Education). Finally, the authors thank the students of ENGR3030U collectively for the use of their DSM models and results.

\section{References}

[1] S.D. Eppinger, D.E. Whitney, R.P. Smith, and D.A. Gebala, Research in Engineering Design, Vol. 6, No. 1, 1994, pp. 1-13.

[2] R.P. Smith and S.D. Eppinger, Management Science, Vol. 43, No. 3, March 1997, pp. 276-293.

[3] R.P. Smith and S.D. Eppinger, Management Science, Vol. 43, No. 8, August 1997, pp. 1104-1120.

[4] P. Cronemyr, A.Ö. Rönnbäck, S.D.Eppinger, Journal of Engineering Design, Vol. 12, No. 3, September 2001, pp. 177-199.

[5] D. Kim, Journal of Engineering Design, Vol. 18, No. 3, June 2001, pp. 265-277.

[6] J. Shiau and X. Li, International Journal of Intelligent Control and Systems, Vol. 12, No. 1, March 2007, pp. 24-36.

[7] Barari, A. and Pop-Iliev, R., Journal of Manufacturing Systems, Vol. 28, Issues 2-3, July 2009, pp. 47-54.

[8] M.S. Pishvaee and S.A. Torabi, Fuzzy Sets and Systems, Accepted April 2010, In Press.

[9] S.G. Lee, K.L. Ong, and L.P. Khoo, Concurrent Engineering, Vol. 12, No. 1, March 2004, pp. 59-66.

[10] P.C. Matthews and C.D.W. Lomas, Journal of Engineering Design (iFirst online article), September 26, 2008, pp. 1-13

(http://www.informaworld.com/smpp/content $\sim \mathrm{db}=\mathrm{all}$ ? content $=10.1080 / 09544820802310909$ ).

[11] G.F. Franklin, J.D. Powell, M. Workman, Digital Control of Dynamic Systems, Third Edition, Addison Wesley Longman, Inc., 1998.

[12] K. Ogata, Discrete-Time Control Systems, second edition, Prentice Hall Inc., 1995. 